Revue d'histoire du XIXe siècle

Société d'histoire de la révolution de 1848 et des

révolutions du XIXe siècle

47 | 2013

Quel est l'avenir du XIX $X^{\mathrm{e}}$ siècle ?

\title{
Modernité équivoque
}

\section{Marc Desgrandchamps}

URL : http://journals.openedition.org/rh19/4543

DOI : $10.4000 /$ rh 19.4543

ISSN : $1777-5329$

Éditeur

La Société de 1848

Édition imprimée

Date de publication : 31 décembre 2013

Pagination : 41-45

ISSN : 1265-1354

Référence électronique

Marc Desgrandchamps, " Modernité équivoque », Revue d'histoire du XIXe siècle [En ligne], 47 | 2013, mis en ligne le 31 décembre 2016, consulté le 19 avril 2019. URL : http://journals.openedition.org/ rh19/4543 : DOI : 10.4000/rh19.4543

Ce document a été généré automatiquement le 19 avril 2019

Tous droits réservés 


\title{
Modernité équivoque
}

\author{
Marc Desgrandchamps
}

1 Dans un texte autobiographique Walter Benjamin déclare que s'il écrit un meilleur allemand que la plupart des écrivains de sa génération, il le doit au fait qu'il n'utilise jamais le mot « je »'. C'est tout le contraire qui va survenir dans les lignes qui suivent où «l'avenir du XIX siècle » sera envisagé par mon «je » au travers de quelques peintres et tableaux, particulièrement l'un d'entre eux, et de la résonance qui est la leur au sein de ma propre pratique.

Le Déjeuner sur l'herbe, c'est par cette peinture vue en reproduction dans une monographie consacrée à Paul Cézanne que le XIXe siècle a surgi pour moi comme un fait tangible et provocant. J'étais très jeune et je crois que cette œuvre de Manet m'est apparue avec la même force, la même inconvenance que lorsqu'elle fut présentée au "Salon des Refusés » de 1863. Il y a là une sorte d'anachronisme car lorsque j'ouvris ce livre, nous étions en 1972. Le sentiment du bizarre éprouvé alors aura plus tard des répercussions dans ma façon d'aborder et de concevoir mon travail pictural.

3 La Liberté guidant le peuple, d'Eugène Delacroix, est un autre tableau dont la découverte au détour des pages d'un manuel scolaire eut peu après les mêmes effets. La violence de représentation liée à la nudité de certaines figures réactiva en moi cette impression du bizarre et de l'étrange. Ici la forme se matérialisait de manière fougueuse, sur fond d'émeute et de révolution. Elle se rattachait à l'Histoire, aux événements. Dans des années 1970 dont la première moitié fut vécue avec l'illusion, quand arrivait le printemps dans certains collèges et lycées, de rejouer un mois de mai mémorable, une telle vision pouvait durablement émouvoir un lycéen sensible aux images et aux représentations. Car il ne s'agissait encore que d'images, la vision des originaux n'étant pas à l'ordre du jour pour cause d'éloignement géographique.

4 Cette manière d'appréhender les œuvres au travers de leur reproduction est évidemment très banale mais elle entraînait une forme d'équivoque, car l'image prenait le dessus sur le bloc de matérialité que représente toute peinture, par son format et sa matière.

5 «Équivoques » était d'ailleurs le titre d'une exposition du musée des Arts Décoratifs qui suscita un certain émoi en 1973, exposition dans laquelle furent visibles pour la première fois depuis que les «impressionnistes» avaient gagné la guerre, un certain nombre 
d'artistes dits «pompiers». Je n'ai pas eu l'occasion de la visiter mais de nombreuses reproductions parurent dans la presse, l'événement étant assez commenté. Je me souviens ne pas avoir compris pourquoi de si jolis tableaux, presque photographiques, s'attiraient des critiques aussi condescendantes et sarcastiques. La reproduction les magnifiait, ce qui pour certains de ces artistes comme Gérôme n'était pas un fait anodin, car j'ai su depuis que ce peintre sculpteur avait une conscience aigüe des avantages commerciaux présentés par la diffusion de ses œuvres. D’une certaine façon il témoignait là d'une ouverture à de nouvelles techniques auxquelles une figure de la modernité comme Cézanne restait indifférent. C'est une des marques de l'ambiguïté, de l'équivoque propre à cette époque.

6 Treize années plus tard, le musée d'Orsay ouvrait en faisant la part belle à ces œuvres, sortant des réserves de nombreuses toiles et sculptures qui n'avaient pas été montrées depuis longtemps. Là aussi certains s'émurent en arguant du fait que l'on ne pouvait tout mélanger, que le goût du public devait être formé, et qu'il ne fallait pas confondre Cabanel et Manet, ce que personne ne songeait à faire. Jeune artiste à ce moment, il me semblait intéressant de revisiter une histoire, un récit moins simplistes que ceux qui avaient cours précédemment. Après tout Bonnat était ami avec Degas, les souvenirs d'Antonin Proust témoignaient du respect de Cabanel pour Manet, et ces artistes respirant finalement le même air, vivant les mêmes temps, il semblait légitime de les montrer ensemble.

7 Cependant, une année ou deux avant l'inauguration d'Orsay, une rétrospective Bouguereau avait déjà marqué les limites de l'exercice. Nous étions plusieurs, en ce début et milieu de la décennie 1980, à pratiquer une peinture figurative qui tentait de réévaluer son histoire, en tâtonnant sans doute avec excès hors des sentiers battus. L'annonce de cette exposition avait été accueillie avec curiosité. La déception fut grande. Le kitsch peut être une valeur contemporaine mais encore faut-il qu'il soit manié avec ironie et conscience, ce qui chez Bouguereau n'était manifestement pas le cas. Seul l'humour involontaire pouvait intéresser à certaines représentations, mais très vite la lassitude venait en contemplant ces accumulations d'angelots accrochés en grappes à des nymphes naïves. Je me rappelle d'un de mes camarades déclarant que c'était de «la peinture gazeuse». La seule chose qui depuis m'ait intrigué chez ce peintre est venue d'un souvenir de Matisse se remémorant sa visite à Bouguereau. Matisse était alors très jeune et il avait trouvé le vieux maître en train de recopier à l'identique un de ses tableaux primé au Salon. Matisse dit en avoir été choqué, ce qui est logique pour un peintre moderne attaché à l'expressivité non reproductible et unique du travail sur la toile. En revanche la fétichisation de l'original pur ayant sombré avec une certaine idée de la modernité, cette pratique de la copie et le détachement qu'elle implique me semble pouvoir entrer en résonnance avec quelques démarches contemporaines qui prennent pour objet la réplique. Ce n'est pas sans rapport avec l'intérêt de Gérôme pour la diffusion photographique de ses œuvres. Par ailleurs on a pu voir récemment qu'un autre grand peintre moderne, Edvard Munch, s'était livré à cette pratique de la copie de ses propres tableaux tout au long de sa vie, ce qui n'est pas sans intriguer chez un artiste censé être associé à l'expressionnisme.

8 Certaines trouvailles hors des fameux sentiers battus s'avérèrent cependant plus stimulantes, ainsi celles de Ferdinand Hodler ou de Max Klinger. Il ne s'agissait pas d'imiter des artistes passés mais plutôt de se servir au sein de l'histoire des formes comme à l'intérieur d'un libre service. Cela s'accompagnait d'une désinvolture assez 
salutaire, désinvolture avec laquelle il était possible de parcourir les allées du musée d'Orsay au cours des années 1980 et même au delà.

Le Déjeuner sur l'herbe demeurait et demeure pour moi une des toiles essentielles au cœur de cet ensemble. Les nus que je réalisais vers 1995 étaient baignés du souvenir de cette toile et de quelques autres figures, comme les Vénus déformées de Ingres passées au crible des Demoiselles d'Avignon et de la revue Playboy.

Pour en rester au Déjeuner, je retrouve dans la formule d'un critique de l'époque, écrivant à propos de Manet «il a le goût corrompu par l'amour du bizarre »" une part de mes motivations. Cette formule se veut négative, mais comme souvent en matière de critique, les termes utilisés peuvent se renverser et apparaître de façon positive, ce n'est là qu'une affaire de point de vue. Le motif étrange de la composition, issu de la Renaissance italienne, sa juxtaposition de l'habillé et du déshabillé, son attention aux détails telle cette grenouille à la base du tableau, son hétérogénéité de surface, ses négligences, ses virtuosités, en font une somme plus stimulante que si le tableau s'incarnait dans une perfection égale et absolue. C'est une peinture qui permet à autrui de faire œuvre, à commencer par de grands exemples comme Picasso et sa série peinte autour de ce thème, ou l'artiste Alain Jacquet qui dans les années 1960 reconstitue cette scène pour en donner une version Pop. C'est un tableau germinateur, peut-être parce qu'il est lui même issu d'un ensemble d'images préexistantes, comme ce dessin perdu de Raphaël gravé par Marcantonio Raimondi. Ces œuvres préexistantes sur lesquelles il est possible de prendre appui pour, ainsi que l'écrivait Louis Althusser, en tirer les réflexions qu'elles contiennent en germe ou ne contiennent pas. Ici on pourrait substituer au mot "réflexions" les termes d'attitudes et de formes.

11 En même temps il me semble qu'il y a chez Manet une sorte de scepticisme que l'on retrouve dans la phrase de Baudelaire, «vous n'êtes que le premier dans la décrépitude de votre art » (lettre à Manet, 11 mai 1865). Ce scepticisme du peintre conscient que son art ne peut plus avoir l'ambition d'évoquer les grands sujets liés à la religion et l'histoire, sinon à la façon du pastiche ou de la caricature, caricature involontaire dans laquelle s'engouffreront la majeure partie des artistes de son temps, fait qu'il restitue cette scène de plein air sur un mode désaccordé. Ce désaccord est l'effet d'un récit impossible, d'une mise en scène dont l'historia chère à Alberti s'est enfuie. "La vérité en peinture ", pour reprendre cette expression cézannienne, se dévoile mieux au travers d'un citron ou d'une simple asperge, que par la volonté de restituer l'exécution d'un empereur du Mexique. On retrouve ce scepticisme, teinté de détachement, dans les premiers tableaux antiquisants d'Edgar Degas. Ils sont souvent inachevés, à l'image de ces Jeunes filles spartiates provoquant des garçons à la lutte, et si Flaubert décrit l'état de tristesse nécessaire pour tenter de ressusciter Carthage, Degas sait qu'il ne peut réanimer Sparte ou Babylone en un siècle où les usines ont remplacé les temples. Il le sait et il le montre en recherchant malgré tout une certaine vérité, qui ne serait pas celle, documentaire et fallacieuse, que l'on trouve chez certains peintres d'histoire. Ainsi de Jean-Léon Gérôme, son contemporain, dont les gladiateurs plagient par anticipation ceux qui évolueront plus tard dans les arènes d'Hollywood. Il ne manque pas un reflet à leur casque. Gérôme sait également qu'il ne peut réanimer Rome, mais à la différence de Degas il ne le montre pas et mime une antiquité en laiton. La vérité de Degas passe par une forme et une matière qui refusent le spectaculaire pour laisser apparaître le pictural en lui-même, détaché de tout enjeu de reconstitution factice. L'équivoque, et nous retrouvons ce mot, demeure dans le fait que le Déjeuner et les Jeunes spartiates sont, malgré un sujet défini comme "prétexte à 
peindre " par Émile Zola, des représentations dont on n'épuise pas le mystère; elles ne sont donc pas uniquement les avant-courriers de la peinture abstraite, comme l'a supposé une vision strictement formaliste et évolutionniste de l'art pour les deux derniers siècles.

Elles témoignent du malaise qui touche alors la représentation, malaise qu'annonce Stendhal un peu plus tôt lorsqu'il écrit que «le sujet ne fait rien au mérite du peintre ", lui concédant juste le rôle des "paroles d'un libretto pour la musique »3. Ce malaise est toujours à l'œuvre aujourd'hui, au sein d'une expérience picturale qui se voudrait dans l'écho du réel, d'un récit ou d'une narration. Elle est contrainte de chercher sa forme propre, sa pertinence, après un $\mathrm{XX}^{\mathrm{e}}$ siècle où dans la résonance des bouleversements historiques, elle a été violentée, brisée et dissoute.

Cela se fait de biais, à la façon dont Degas imagine Sparte, et Manet l'illusion d'un sous bois dans la lumière d'un studio. Les peintures sont des manières de trace ou d'évocation, mais ne s'identifient pas à la chose représentée. Ces traces peuvent être brillantes, lumineuses, à l'image des tableaux observés dans ce texte. Lorsque je les découvris adolescent, ils me surprirent par ce que je ressentis comme une transgression, une érotisation des figures, dans mon quotidien assez morne d'alors. Je n'ai jamais oublié cette interprétation première, intuitive, d'où mes réticences ultérieures à épouser le point de vue formaliste à propos d'un tableau comme Le Déjeuner. Sa complexité dépasse ce simple schéma, et même si l'affirmation de la peinture comme matière spécifique y est évidemment présente, il ne se cantonne pas à être une pure surface. Il est aussi une scène énigmatique, bâtie sur un ensemble de réminiscences, et pour reprendre les mots de George Kubler, le possible objet premier d'une série picturale qui ne renoncerait pas à l'ambition d'être une figure du monde.

14 L'œuvre de Manet continue à me surprendre. Récemment j'ai remarqué le liseré vert délimitant le haut de la cuisse d'Olympia de la tenture plane et sombre qui lui sert de fond. Cette ligne verte, fortement nuancée, m'a saisi alors qu'une nouvelle fois je me penchais sur cette toile, autre chef-d'œuvre du peintre. On ne regarde jamais assez attentivement. Il faut revoir les tableaux comme on relit les livres. Ils se transforment avec notre regard.

Le XIX ${ }^{e}$ siècle a été le temps où la modernité s'est fondée, avec dans son sillage le mélange d'affirmations et de doutes qui constituent son apport. Quelques tableaux en témoignent. Ils agissent comme des stimulants pour travailler et faire œuvre.

\section{NOTES}

1. "Chronique berlinoise ", in Écrits autobiographiques, traduit de l'allemand par Christophe Jouanlanne et Jean-François Poirier, coll. «Détroits », Paris, Christian Bourgois Éditeur, 1990, p. 260.

2. Ernest Chesneau, L'art et les artistes modernes en France et en Angleterre, Paris, 1864, p. 189.

3. Stendhal, Promenades dans Rome, « 28 août 1827 ». 


\section{AUTEUR}

\section{MARC DESGRANDCHAMPS}

Marc Desgrandchamps est peintre ; une rétrospective de son œuvre a été exposée au Musée d'art moderne de la Ville de Paris en 2011 